\title{
LIPSCHITZIAN PARAMETERIZATIONS AND EXISTENCE OF MINIMA IN THE CALCULUS OF VARIATIONS
}

\section{GEORGE M. EWING}

This note exhibits a brief and relatively elementary approach which the author has used when time and other exigencies precluded a more conventional development. The reader is referred to the recent paper of Cesari [1], particularly $\S \S 8,10,11$.

Let $x$ be a continuous rectifiable mapping of a closed interval $[a, b]$ into a fixed bounded closed subset $A$ of the $E_{n}$ and let $\left[t_{i-1}, t_{i}\right]$ denote a general subinterval of $[a, b]$ under a partition. Let $f$ be a real function of $(x, r) \in A \times E_{n}$ subject to the conditions

I. $f$ is continuous in $(x, r)$,

II. $f(x, k r)=k f(x, r), k \geqq 0$,

III. $f(x, r)>0, r \neq 0$.

Letting the norm of the partition tend to 0 , one then defines the Weierstrass integral denoted here by $W[x ; a, b ; f]$ as the limit,

$$
\lim \sum f\left[x\left(T_{i}\right), x\left(t_{i}\right)-x\left(t_{i-1}\right)\right] .
$$

The limit exists independently of the choice of $T_{i} \in\left[t_{i-1}, t_{i}\right]$ and if $y(u), u \in[c, d]$ is Fréchet-equivalent $[1$, p. 494] to $x(t), t \in[a, b]$ then $[2$, p. 679],

$$
W[y ; c, d ; f]=W[x ; a, b ; f] .
$$

We require further of $f$ that for each admissible $x$ there exists on each subinterval $\left[t, t^{\prime}\right]$ of the parameter interval a number $T$ such that

IV. $f\left[x(T), x\left(t^{\prime}\right)-x(t)\right] \leqq W\left[x ; t, t^{\prime} ; f\right]+\left|x\left(t^{\prime}\right)-x(t)\right|^{2}$.

Mapping $x$ is termed $f$-Lipschitzian (abbreviated $f L$ ) on $[a, b]$ if there is a constant $k$ and on each subinterval $\left[t, t^{\prime}\right]$ a point $T$ such that

$$
f\left[x(T), x\left(t^{\prime}\right)-x(t)\right] \leqq k\left|t^{\prime}-t\right| .
$$

Lemma 1. A necessary and sufficient condition for $x$ to be fL on $[a, b]$ is that $x$ be Lipschitzian on $[a, b]$.

Proof. Using conditions I, III, observe that there exist positive constants $m, M$, such that for $x \in A$ and $|r|=1, m \leqq f(x, r) \leqq M$. This holds for the unit vector $r /|r|$ when $r \neq 0$, while $f(x, 0)=0$ from II. The stated result then follows from II and the above inequalities.

Received by the editors March 5, 1959 and, in revised form, March 23, 1959. 
Lemma 2. A necessary and sufficient condition for $x$ to be fL on $[a, b]$ is that there exist a constant $k$ and on each subinterval $\left[t, t^{\prime}\right]$ of $[a, b] a$ number $T$ such that

$$
f\left[x(T), x\left(t^{\prime}\right)-x(t)\right] \leqq k\left|t^{\prime}-t\right|+\left|x\left(t^{\prime}\right)-x(t)\right|^{2} .
$$

Clearly (3) implies (4). Given (4) let $\left[t, t^{\prime}\right]$ now be fixed and let $\pi$ be a partition of $\left[t, t^{\prime}\right]$. Applying (4) separately to the subintervals, adding results, and considering limits as norm $\pi$ tends to 0 with reference to (1), we find that $W\left[x ; t, t^{\prime} ; f\right] \leqq k\left|t^{\prime}-t\right|$. However, for a

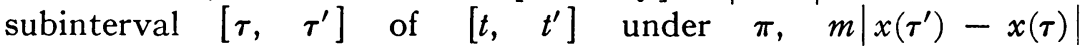
$\leqq f\left[x(T), x\left(\tau^{\prime}\right)-x(\tau)\right]$; hence from (1) applied both to the given $f$ and to the function $\lambda, \lambda(x, r)=|r|$, it follows that $m W\left[x ; t, t^{\prime} ; \lambda\right]$ $\leqq W\left[x ; t, t^{\prime} ; f\right]$. The integral on the left is simply the length of the mapping. It follows that $m\left|x\left(t^{\prime}\right)-x(t)\right| \leqq k\left|t^{\prime}-t\right|$; hence that $x$ is Lipschitzian with constant $k / m$.

Consider the class $K$ of all admissible mappings $x$ such that each $x \in K$ has the unit parameter-interval, satisfies (4) for some $k$, and is Fréchet-equivalent to each other $x \in K$.

Lemma 3. The set of numbers $k$ associated with mappings $x$ of $K$ has a minimum, viz. $\min k=J(C, f)$ denoting the common value of all integrals (2) for $x, y \in K$.

PROOF. The equivalence class $K$ includes the particular parameterization $\xi$ in terms of reduced $J$-length, i.e. the mapping $\xi$ such that for each subinterval $\left[t, t^{\prime}\right]$ of $[0,1]$

$$
W\left[\xi ; t, t^{\prime} ; f\right]=\left(t^{\prime}-t\right) J(C, f) .
$$

Existence of $\xi$ can be established along the lines of $[1, \S 10]$. If one accepts the existence of at least one light parameterization, e.g. that in terms of reduced length $t / L(C)$, then $\xi$ is obtained quickly from condition III on $f$ and the nature of strictly increasing functions.

Since any mapping $x \in K$ satisfies (4) on the unit interval, we find by an argument used in the proof of Lemma 2 that $W[x ; 0,1 ; f] \leqq k$; hence that $J(C, f) \leqq k_{0}$, the infimum of values $k$ for which (4) holds on the class $K$. Applying IV to the particular mapping $\xi$ and using (5) we see that $J(C, f)$ is a particular $k$ for which (4) holds on $K$. Thus $k_{0} \leqq J(C, f)$ so that actually the equality holds and $k_{0}$ being realized through $\xi$ is a minimum.

Existence Theorem. Let $X$ denote the class of all admissible parameterizations $x$ whose graphs join disjoint closed subsets of $A$. If $X$ is nonempty and $f$ has properties I, II, III, IV there exists $x_{0} \in X$ minimizing $W$ in $X$. 
Proof. Denote the infimum of $W$ on $X$ by $k_{0}$. Let $x_{v}, \nu=1,2, \cdots$ be a sequence on $X$, which in the light of Lemma 3 can be chosen so that $x_{\nu}$ has the unit parameter interval and satisfies (4) with constant $k_{\nu}, \lim k_{\nu}=k_{0}$. With the aid of Lemmas 1,2, we see that the $x_{\nu}$ are all Lipschitzian with a common constant; hence that they are equicontinuous on $[0,1]$ and by Ascoli's theorem [3, p. 336] we can suppose sequence $x_{v}$ to have been chosen so as to converge uniformly on $[0,1]$ to a limit $x_{0}$. Given a subinterval $\left[t, t^{\prime}\right]$ of $[0,1]$, then to each $\nu$ corresponds a number $T_{\nu} \in\left[t, t^{\prime}\right]$ such that (4) holds with $x_{\nu}, k_{\nu}, T_{\nu}$. Thus a suitable subsequence of $x_{\nu}$ again denoted by $x_{\nu}$ has the property that $T_{\nu}$ converges to $T_{0} \in\left[t, t^{\prime}\right]$. It follows that (4) holds for $x_{0}, k_{0}, T_{0}$ since otherwise (4) in $x_{\nu}, k_{\nu}, T_{\nu}$ is false for sufficiently large $\nu$. It follows from (4) that $W\left[x_{0} ; 0,1 ; f\right] \leqq k_{0}$; hence by the definition of $k_{0}$ that equality must hold.

Certain types of side conditions could have been included in the definition of class $X$. The theorem can be rephrased in terms of Fréchet curves.

The writer has not been able to determine the relation between convexity of $f$ in its second argument and the regularity condition IV. If $f(x, r)=\phi(x) g(r)$ with $g$ convex in $r$ then IV holds in strict form, i.e. without the second term on the right. If set $A$ has an interior point $b$, if $\phi(x)=$ constant, and if $g\left(r_{1}+r_{2}\right)>g\left(r_{1}\right)+g\left(r_{2}\right)$ then consideration of a short broken line issuing from $b$ whose two segments have respective directions $r_{1}, r_{2}$, leads to a denial of the strict form of IV. However, the trivial subcase in which $A$ is a segment and $g$ is not convex does satisfy the strict form of IV.

Thus the class of problems covered by the theorem intersects nonvacuously with that included under theorems based on convexity and semi-continuity but is not included in the latter and probably vice versa.

Second terms on the right in conditions IV on $f$ and (4) on $x$ can be both replaced by any other function of the difference vector whose sum on subintervals of a partition tends to zero with the norm.

\section{REFERENCES}

1. L. Cesari, Rectifiable curves and the Weierstrass integral, Amer. Math. Monthly vol. 65 (1958) pp. 485-500.

2. G. M. Ewing, Variation problems formulated in terms of the Weierstrass integral, Duke Math. J. vol. 14 (1947) pp. 675-687.

3. E. J. McShane, Integration, Princeton University Press, 1944.

UNIVERSITY OF OKLAHOMA AND

The U. S. Army Artillery and Missile School, Fort Sill, Oklahoma 